\title{
Comprensión de la noción de función y la articulación de los registros semióticos que la representan entre estudiantes que ingresan a un programa de ingeniería
}

\author{
Understanding the notion of function and articulation of semiotic records that \\ represent between students entering a program Engineering
}

\section{Compreender a noção de função e articulação de registros semióticos que representam entre alunos que entram num programa de Engenharia}

\author{
Raúl Prada-Núñez ${ }^{1}$ \\ Cesar Augusto Hernández-Suárez² \\ Pastor Ramírez-Leal ${ }^{3}$
}

Fecha de recepción: mayo 2016

Fecha de aceptación: junio 2016

Para citar este artículo: Prada-Núñez, R.; Hernández-Suárez, C.A. y Ramírez-Leal, P. (2016). Comprensión de la noción de función y la articulación de los registros semióticos que la representan entre estudiantes que ingresan a un programa de ingeniería. Revista Científica, 25, 188-205. Doi: 10.14483 /udistrital.jour.RC.2016.25.a3

\section{Resumen}

En el presente estudio se propone evaluar la comprensión de la noción de función y la capacidad de articular distintos registros semióticos para su representación por parte de los estudiantes de nuevo ingreso en la carrera universitaria en Colombia. Se toma como contexto inmediato la Facultad de Ingeniería de una universidad pública. Epistemológicamente, el estudio se basa en el interaccionismo simbólico mediante el análisis de los significados que los estudiantes atribuyen a este concepto matemático cuando abordan la resolución de problemas. La metodología adoptada, en consecuencia, es cualitativa y se hace uso de la codificación teórica. Para el análisis de los resultados se ha aplicado la teoría fundamentada con un enfoque estructurado. La información que genera este estudio corresponde a una prueba en la que se mostró a los estudiantes dos representaciones gráficas con la intención de que identificaran cuál de ellas constituía una función, además de lo cual debían argumentar su respuesta. En total se analizan 86 argumentos alrededor del concepto. El análisis de datos se hizo a través del software Atlas.ti 7.0. El sistema de categorías emergente permite vislumbrar los siguientes hallazgos: deficiencias conceptuales, diversidad en aproximaciones conceptuales, referentes conceptuales, representaciones semióticas y, por último, destacan las distintas variaciones conceptuales.

Keywords: representaciones semióticas, variaciones conceptuales, registros semióticos

\footnotetext{
Abstract
}

This study aims to assess the understanding of the notion of function and the ability to articulate different semiotic registers for representation by new 
students in the university in Colombia. Immediate context is taken as the Faculty of Engineering at a public university. Epistemologically the study is based on symbolic interaction by analyzing the meanings that students attributed to this mathematical concept when addressing problem solving. The methodology, therefore, is qualitatively and use of theoretical coding is done. For analysis of the results it has been applied grounded theory with a structured approach. The information generated by this study corresponds to a test that showed students two graphic representations with the intent to identify which of them was a function, besides which should argue their response. Altogether 86 arguments around the concept are analyzed. Data analysis was done through atlas.ti 7.0 software. The system allows a glimpse of emerging categories the following findings: conceptual deficiencies, diversity in conceptual approaches, conceptual referents, semiotic representations, and finally highlight the various conceptual variations.

Palabras Clave: semiotic representations, conceptual variations, semiotic registers.

\section{Resumo}

Este estudo tem como objetivo avaliar a compreensão da noção de função e a capacidade de articular diferentes registros semióticos para a representação de novos alunos na universidade na Colômbia. contexto imediato é tida como a Faculdade de Engenharia em uma universidade pública. Epistemologicamente o estudo baseia-se na interação simbólica através da análise dos significados que os alunos atribuídos a este conceito matemático, ao abordar a resolução de problemas. A metodologia, por conseguinte, é qualitativamente e utilização de codificação teórica é feito. Para análise dos resultados foi aplicada a teoria aterrado com uma abordagem estruturada. As informações geradas por este estudo corresponde a um teste que mostrou aos alunos duas representações gráficas com a intenção de identificar qual deles era uma função, além de que deve discutir sua resposta. No total, 86 argumentos em torno do conceito são analisados. A análise dos dados foi feita através de software atlas.ti 7.0. 0 sistema permite um vislumbre do emergente categorias as seguintes constatações: deficiências conceituais, diversidade de abordagens conceituais, referentes conceituais, representações semióticas e, finalmente, realçar as diversas variações conceituais.

Palavras chave: representações semióticas, as variações conceituais, registros semióticos

\section{Marco Conceptual}

Un problema severo y recurrente en el quehacer académico de profesores, y también para la propia investigación en educación matemática, consiste en que un considerable número de estudiantes no alcanza el nivel esperado en las asignaturas de matemáticas. Con este panorama, la intención de esta investigación consiste en el abordaje de un problema específico, referido a la comprensión de la noción de función entre un grupo estudiantes universitarios y en la capacidad que poseen para articular diversos registros semióticos en los que puede expresarse con vistas al diseño e instrumentación de estrategias para su apropiada enseñanza y aplicación. Dentro de este propósito quedan incluidos las dos grandes complicaciones contra los que chocan los estudiantes: la comprensión de los conceptos matemáticos y la capacidad de aplicarlos a distintos eventos y situaciones. Por otra parte, resulta obvio que analizar el problema del aprendizaje de las matemáticas desde un enfoque global escapa a los límites y posibilidades del investigador, pero el acopio de un conjunto de investigaciones seriadas con toda seguridad permitirá una comprensión general del problema.

Desde otra perspectiva, el concepto de función y la comprensión de su utilidad por el estudiante resultan paradigmáticos dentro de los problemas de aprendizaje de las matemáticas, pues se trata de una noción relativamente sencilla que los estudiantes, sin embargo, muestran significativas confusiones y limitaciones. Esta misma situación se encuentra en la comprensión de otros conceptos del cálculo, por lo que la investigación sobre este punto específico puede también arrojar luz, por analogía, sobre otros 
problemas de la misma naturaleza. El concepto de función, en efecto, se muestra en apariencia sencillo y de fácil entendimiento, pero investigaciones llevadas a término sobre este respecto demuestran que conlleva frecuentemente dificultades de un cierto grado para su entendimiento cabal (Planchart, 2002). En la práctica educativa, la aprehensión de este concepto ha resultado de tal complejidad que desarrollar un estudio de esta problemática adquiere interés de investigación.

En ocasiones se ha observado que los estudiantes, después de estudiar el concepto y los subconceptos de función, alcanzan una comprensión que a veces se limita al uso de una regla para probar cuándo una relación constituye una función; en otros, a la sola evaluación de funciones en el contexto algebraico. Como lo cita Artigue (1995), la enseñanza de los conceptos fundamentales del cálculo es fuente generadora de problemas. En efecto, los docentes ofrecen a sus estudiantes herramientas mecánicas para realizar algunos procesos, como determinar si una expresión algebraica es una función, o también la manera de hallar un límite al infinito o determinar un máximo relativo a través del proceso de derivación. Sin embargo, el hecho de que el estudiante realice los procesos mecánicos de forma más o menos correcta no implica que haya alcanzado una comprensión satisfactoria de dichos conceptos, muy seguramente debido a que la enseñanza universitaria tradicional tiende a favorecer la práctica algorítmica y algebraica del cálculo, reduciendo los procesos evaluativos no al entendimiento y aplicación de conceptos, sino a la réplica mecánica de ciertos protocolos de solución. Este grado básico de comprensión, con el que a veces los propios docentes se conforman, restringe la potencialidad del aprendizaje y el logro del objeto matemático, ya que es necesario que el estudiante se apropie del concepto y sea capaz de representarlo de distintos modos; de lo contrario, afrontará dificultades al tratar de entender y transferir esta y otras nociones matemáticas a otros temas y problemas de precálculo y cálculo diferencial e integral.

De lo expuesto queda claro que entre los estudiantes existen dificultades en torno a conceptos de precálculo y cálculo, esto como posible efecto de la aplicación de un sistema tradicional de enseñanza que ha favorecido la utilización de unos registros más que otros. Por esta razón, es necesario evaluar científicamente el problema y establecer una tipología de errores y falta de destrezas en el manejo y aplicación de las nociones fundamentales de cálculo a fin de diseñar estrategias pedagógicas de intervención lo más cercanas posible a la superación de estos escollos.

Debido a la imposibilidad de abarcar en una sola investigación todos los problemas derivados del inadecuado manejo y relación entre los conceptos relativos al cálculo, esta investigación se concentra en el concepto de función. El trabajo se emprende, además, desde la óptica de la aplicación de las representaciones semióticas. La investigación se centra en el concepto de función y toma como referencia la articulación de los trabajos de diversos investigadores, especialmente los de Duval (1988, 1993, 2006); Hitt (1994, 1998, 2000, 2003a, 2003b, 2003c) y Vergnaud (1983, 1990, 2007)

Así mismo se consideran algunos trabajos investigativos desarrollados en los últimos años en el contexto colombiano, entre los cuales se destacan los estudios de Garzón (2015), que parte del análisis de tres aspectos sustanciales: i) el proceso de modelación de situaciones y problemas en contextos reales para el desarrollo de competencias STEM (ciencias, tecnología, ingeniería y matemáticas, por sus siglas en inglés), ii) el enfoque teórico de Duval sobre las diversas representaciones semióticas de este concepto, y iii) su desarrollo histórico epistemológico.

También se destaca el trabajo de Sánchez, Martínez y Coronado (2015) quienes hacen una caracterización de la competencia matemática representar, asociada al objeto matemático función lineal cuyo resultado más relevante fue el empleo 
de diferentes sistemas de representación semiótica (íconos, tablas, verbal) en el que el componente matemático y el uso social de las matemáticas se asoció al predominio de la representación icónica para la situación problémica propuesta.

En la investigación de García (2013), se planteó la idea de función como modelo matemático, es decir, un objeto que atrapa la variación y el cambio, además de las diversas interpretaciones y relaciones que se pueden hacer de la representación tabular, algebraica, gráfica y el lenguaje natural que en conjunto forman los registros de representación que se abordan en el trabajo.

Por último, el trabajo de Ospina (2012) indagó sobre las representaciones semióticas del concepto de función lineal, tomando como referente investigativo el enfoque semiótico de Duval mediante el análisis de las producciones de los estudiantes, desde las cuales se exploran los registros en los que hacen transformaciones cognitivas (tratamiento y conversión), la forma en que las hacen y la coordinación de diferentes registros de representación del concepto matemático función lineal, siendo la conversión de registro la actividad que centra la atención de la investigación.

Entre otras investigaciones relacionadas con las representaciones semióticas, se encuentra la desarrollada por Rojas (2012, 2014) orientada por D'Amore, que buscó documentar el fenómeno relacionado con la articulación y el cambio de sentidos asignados a representaciones semióticas de un mismo objeto matemático, obtenidas mediante tratamiento. El autor pone en evidencia que las transformaciones de tratamiento pueden ser tan complejas como las de conversión y, por tanto, ser fuente de dificultades en los procesos de comprensión de la matemática escolar.

Por lo mencionado anteriormente y sin ser exhaustivos, el propósito de esta investigación consiste en evaluar la capacidad de comprender y articular los diversos registros de representación semiótica en los estudiantes de nuevo ingreso a los programas académicos de ingeniería en torno a la noción de función.

\section{Marco de Referencia}

\section{La noción de función}

El concepto de función resulta fundamental para el entendimiento de las matemáticas a cualquier nivel educativo, sobre todo en el estudio del cálculo en la educación superior (Farfán y García, 2005). Por esta razón, el conocimiento de las funciones y el modo en que se comprenden y se articulan ha sido el foco de numerosas investigaciones, por mucho tiempo y desde diferentes perspectivas. Las investigaciones que se han llevado a término se han centrado las distintas orientaciones y dificultades alrededor del estudio del concepto de función (Ferrari, 2001): la imagen y definición del concepto (Vinner, 1992, 2002; Tall, 1992, 2002; Eisenberg, 2002), la teoría APOE (Dubinsky, 2002), la dialéctica herramienta-objeto y juegos de contextos (Douady, 1986, 1995, 1996), los obstáculos epistemológicos y actos de entendimiento (Sierpinska, 1992), el pensamiento y leguaje variacional (Cantoral y Farfán, 1998; Farfán, 1997) y la articulación de registros y representaciones semióticas (Artigue, 1995; Duval, 1988, 1993), entre otras muchas. Por la importancia que tiene su punto de vista para esta investigación se destacan por separado las investigaciones sobre funciones centradas en visualización de Hitt (1994, 1998, 2003a, 2003b, 2003c) entre otros.

Las cuatro definiciones de la noción de función más comunes que se han presentado a lo largo del siglo XX son las siguientes (Hitt y Torres, 1994):

Función en términos de variable: Una función es una variable relacionada con otra variable tal que a cada valor de la última le corresponde únicamente un valor de la primera.

Función en términos de conjunto de parejas ordenadas: Una función es un conjunto de pares ordenados, dos de los cuales tienen la misma primera componente.

Función en términos de regla de correspondencia: Una función $\mathrm{f}$ de un conjunto $\mathrm{A}$ a un conjunto $\mathrm{B}$ 
es una regla de correspondencia que asigna a cada $x$ de cierto subconjunto $D$ de $A$ un elemento determinado de manera única $f(x)$ de $B$. En esta definición queda incluido el concepto anterior y es la más generalmente aceptada en la actualidad.

Función en ambiente logo: Una función es un procedimiento $\mathrm{P}$ que tiene la propiedad de que cualesquiera dos apelaciones a P con las mismas entradas producen las mismas salidas.

De estas concepciones de función, la más comúnmente aceptada es la formulada en términos de regla de correspondencia, y es la que mayormente se emplea en la enseñanza del cálculo. En efecto, la reforma de las matemáticas en la década de 1960 impulsó la presentación de la disciplina en términos más formales, en los que la notación conjuntista predominaba. A los estudiantes se les enseñaban definiciones equivalentes a la anterior. Los problemas de aprendizaje de las matemáticas provocados por este acercamiento en la enseñanza pronto emergieron. En un nuevo intento por conciliar formalidad y enseñanza, se presentó a los estudiantes una definición de función que, como se ha comentado antes, hace énfasis en la idea de correspondencia:

Una función $f$ de un conjunto $X$ a un conjunto $Y$ es una regla que asocia a cada elemento $x$ de $X$ un único elemento y de $\mathrm{Y}$. El elemento y se Ilama la imagen de $x$ bajo $f y$ se denota por $f(x)$. El conjunto $X$ se llama dominio de la función. El rango de la función consta de todas las imágenes de los elementos de X.

Representación semiótica del concepto función y variación de registros. Esta investigación se ha enfocado en el marco de la construcción del conocimiento basado en los sistemas de representación, por lo que resulta necesario ahora considerar las distintas representaciones semióticas que pueden configurar esta noción y la manera en que se articulan entre sí. Investigadores como Kaput (1998), Duval (1998), Hitt (2000, 2003a), y otros muchos, han incorporado los sistemas de representación como herramienta de análisis de los procesos de aprendizaje.

Duval (1998, 1999), por su parte, profundizó en el estudio de las representaciones circunscribiéndolas al campo de la semiótica. Según el autor, los sistemas de representación semiótica poseen una serie de características particulares que permiten sostener la conceptualización en la matemática; entre tales características se encuentra la de su utilidad no solo en la comunicación de las nociones, sino además como un elemento importante de la actividad cognitiva misma. Duval (1998, p. 176) reconoce la importancia a las representaciones cuando afirma que "el funcionamiento cognitivo del pensamiento humano se revela como inseparable de la existencia de una diversidad de registros semióticos de representación". Señala, además, que las representaciones semióticas deben cumplir las siguientes funciones: a) la función de comunicación (intercambio social), b) objetivación (toma de conciencia) y c) tratamiento (manipulación de la información). Siempre según este autor, un registro semiótico de representación está condicionado a que permita tres actividades cognitivas fundamentales: a) la formación de una representación identificable, sea esta una frase, dibujo, fórmula escrita, esquema, etc. Esta actividad comprende una selección de rasgos y datos que se pueden representar, que responden a reglas que permiten asegurar las condiciones de identificación y ofrezcan la posibilidad de su utilización en otra actividad cognitiva; b) el tratamiento de una representación, lo cual significa la transformación de la representación en el mismo registro; y c) la conversión de una representación, lo que supone la transformación de una representación de un registro a otro manteniendo la totalidad o parte de la representación inicial.

Si se concibe el concepto de función en el marco teórico de las representaciones semióticas se observan las diferentes representaciones que corresponden a este concepto (tabla 1). 
Tabla 1. Descripción de los tipos de representación semiótica.

\begin{tabular}{ll}
\hline $\begin{array}{c}\text { Tipo de } \\
\text { representación }\end{array}$ & \multicolumn{1}{c}{ Característica } \\
\hline $\begin{array}{l}\text { Lenguaje } \\
\text { natural }\end{array}$ & $\begin{array}{l}\text { Representación que utiliza expresiones } \\
\text { y palabras propias de la lengua, como, } \\
\text { por ejemplo, el cuadrado de un número. }\end{array}$ \\
\hline \multirow{2}{*}{ Tablas } & $\begin{array}{l}\text { Representación horizontal o vertical en } \\
\text { la que se identifican valores asociados } \\
\text { de la variable independiente y } \\
\text { dependiente. }\end{array}$ \\
\hline Gráficas & $\begin{array}{l}\text { Representación de tipo visual que } \\
\text { se apoya en los conceptos de plano } \\
\text { cartesiano, par ordenado y otros del } \\
\text { mismo estilo. }\end{array}$ \\
\hline Fórmulas & $\begin{array}{l}\text { Representación de tipo algebraico en } \\
\text { la que se visualiza la expresión que } \\
\text { relaciona las variables } x \text { y y. }\end{array}$ \\
\hline
\end{tabular}

Fuente: elaboración propia.

Cada modalidad tiene sus propiedades, reglas y significación; en cada una de ellas puede haber transformaciones y se pueden producir traslaciones de una a otra. En algunos estudios se ha señalado que las traslaciones del sistema gráfico a las representaciones algebraicas son las que causan mayores dificultades (Ruthven, 1990; Duval, 1998). Estos problemas podrían superarse si se reconociera la importancia de las reglas de correspondencia semiótica y se establecieran las coordinaciones en distintas direcciones (por ejemplo, si de lo simbólico se va al registro gráfico o de la representación gráfica a la escritura de la respectiva expresión algebraica). La coordinación de los diferentes registros de representaciones aparece como una condición fundamental para el aprendizaje en aquellas disciplinas donde los datos son representaciones semióticas (Duval, 1998).

\section{Teoría de los campos conceptuales}

La teoría de los campos conceptuales se halla dentro de las teorías cognitivas, esto es, las que explican los mecanismos mediante los cuales se construyen y relacionan tanto el conocimiento cognitivo como competencias complejas, particularmente aquellas implicadas en las ciencias y en las técnicas. Parte de la idea de que la adquisición y comprensión del conocimiento complejo requiere de un proceso de maduración de los conceptos, que se adquiere no solo con el transcurso del tiempo sino además con su consideración y estudio desde distintas perspectivas. Todo saber forma parte de un sistema muldimensional; el aprendizaje, en consecuencia, no se produce por el conocimiento de temas aislados, sino por relaciones entre conceptos pertenecientes a un campo. A medida que se analizan y relacionen los temas y conceptos pertenecientes a un campo de conocimiento o área del saber; tales conceptos son comprendidos cada vez mejor. La perspectiva de esta teoría es, por tanto, desarroIlista. Vergnaud (1990), principal ponente de esta teoría, señala que un campo conceptual consiste en un conjunto de problemas y situaciones cuyo tratamiento requiere conceptos, procedimientos $y$ representaciones de tipos diferentes, pero íntimamente relacionados; dicho de otro modo, se trata de un conjunto de situaciones cuyo dominio requiere, a su vez, el dominio de varios conceptos de naturaleza distinta pero afín. Un ejemplo claro es el de las estructuras multiplicativas, que implica todas las situaciones que pueden ser analizadas como problemas de proporciones simples y múltiples para los cuales se necesita una multiplicación, una división o una combinación de ambos procedimientos. En la comprensión de la multiplicación están involucrados varios tipos de conceptos matemáticos, entre los que se encuentran la función lineal, la función no lineal, el espacio vectorial, el análisis dimensional, la fracción o los números racionales. La comprensión plena de las estructuras u operaciones multiplicativas requiere un tipo de pensamiento complejo, que se desarroIla en el tiempo y a través del dominio de una serie de conceptos.

Los fundamentos de la teoría de los campos conceptuales de Vergnaud (1983, p. 393) son tres: a) un concepto no se forma dentro de un solo tipo de situaciones; b) una situación no se analiza con un solo concepto; c) la construcción y apropiación 
de todas las propiedades asociadas a un concepto o a una situación requiere de tiempo y de distintas aproximaciones bajo perspectivas también diferentes, incluidos los errores y fallos.

En relación al término concepto, nuclear en su teoría, Vergnaud considera tres conjuntos, a saber: a) un conjunto de situaciones que dan sentido al concepto; b) un conjunto de invariantes (objetos, propiedades y relaciones) sobre las cuales reposa la operacionalidad del concepto, o un conjunto de invariantes que pueden ser reconocidos y usados por los sujetos para analizar y dominar las situaciones del primer conjunto; y c) un conjunto de representaciones simbólicas (lenguaje natural, gráficos o diagramas, sentencias formales, entre otros) que pueden ser utilizadas para indicar y representar esos invariantes $y$, consecuentemente, representar y manejar las situaciones y los procedimientos (Cf. Moreira, 2002). Eso implica que para estudiar el desarrollo y el uso de un concepto, a lo largo del aprendizaje o de su utilización, es necesario considerar esos tres conjuntos simultáneamente, y que el dominio de un campo conceptual requiere a su vez el dominio de una variedad de conceptos de distinta naturaleza.

\section{Metodología}

El diseño del estudio es de naturaleza cualitativa y se corresponde con el enfoque de la teoría fundamentada (Glasser y Strauss, 1967) y del interaccionismo simbólico (Blumer (1992). Se ha elegido como estrategia central del análisis la codificación teórica (Flick, 2004), por lo que el enfoque del análisis es de carácter interpretativo.

La codificación teórica, en el marco de la teoría fundamentada, permite llevar a cabo el análisis inductivo propio de los procesos de análisis cualitativo, cada uno de los significados otorgados por los participantes a los ítems expuestos, pasan a ser el insumo de interpretación para los investigadores con fundamento en los referentes teóricos.

Bajo esta propuesta se propone a los participantes la evaluación de ejercicios prácticos relacionados con la identificación gráfica y conceptual de funciones. A partir de sus respuestas se extraen los argumentos que han dado sobre los conceptos presentados y se interpretan teóricamente los significados otorgados por los participantes a los elementos de las funciones propuestas.

Desde un punto de vista más amplio, se trata de un diseño de campo, ya que la información ha sido recolectada directamente de los estudiantes, considerados como información primaria (Echevarría, 2005). A partir de los argumentos dados por los estudiantes se pretende realizar una aproximación descriptiva a los elementos teóricos que se encuentran alrededor de la noción del concepto de función siguiendo las etapas de: observación, categorización y asociación propuestos por Carlile y Christense (2011).

\section{Participantes y contexto}

La población objeto de estudio está formada por los estudiantes de nuevo ingreso de la Facultad de Ingeniería de la Universidad Francisco de Paula Santander (UFPS), en su sede de Cúcuta, que está integrada por siete programas académicos de ingeniería: Sistemas, Civil, Industrial, Mecánica, Electrónica, Minas y Electromecánica. En total, participaron 79 estudiantes, con edades comprendidas entre 16 y 21 , de los cuales el $60 \%$ se ubican en el rango entre 17 y 18 años, y distribuidos en dos programas académicos, de Ingeniería de Sistemas (46,8\%) e Ingeniería Electromecánica (53,2\%). El 86,1\% corresponde a estudiantes de género masculino que se graduaron de la educación media entre los años 2010 y 2014, correspondiendo el $62 \%$ a personas graduadas en 2014 . Adicionalmente, el $87 \%$ de los estudiantes de la muestra proviene de instituciones educativas de carácter público y de ellos el 70 \% corresponden a estratos 1 y 2, lo cual se relaciona con el perfil de ingreso del estudiantado de la UFPS (descriptivos obtenidos con la utilización del statgraphics centurion XVII). 
La selección de la muestra, fue realizada mediante muestreo no probabilístico, con empleo de la técnica de muestreo intencional; ello, porque se quiso indagar si existían diferencias académicas entre los estudiantes de nuevo ingreso a dos tipos de programas académicos: uno con acreditación de alta calidad y el otro con registro calificado, condiciones de calidad definidas por el Consejo Nacional de Acreditación (CNA) entidad adscrita al Sistema Nacional de Acreditación del MEN. Se optó por trabajar con estudiantes del grupo A, debido a que no hay entre ellos estudiantes repitentes, lo que constituiría un factor de ruido en el experimento. En este tipo de grupo, además, los estudiantes casi siempre poseen características similares en aspectos como edad, año de egreso de la educación media, lo cual determina cierta homogeneidad en relación con ciertos factores sociales que pueden incidir en el rendimiento académico y llegar por ello incluso a sesgar los resultados.

\section{Instrumentos}

Se diseñó un instrumento de nueve ítems que muestran diversos registros de representación alrededor del concepto de función. La información que recoge este artículo corresponde al primer ítem, que mostró a los estudiantes dos representaciones gráficas con la intensión de que identificaran cuál de ellas corresponde a una función, debiendo argumentar, su respuesta.

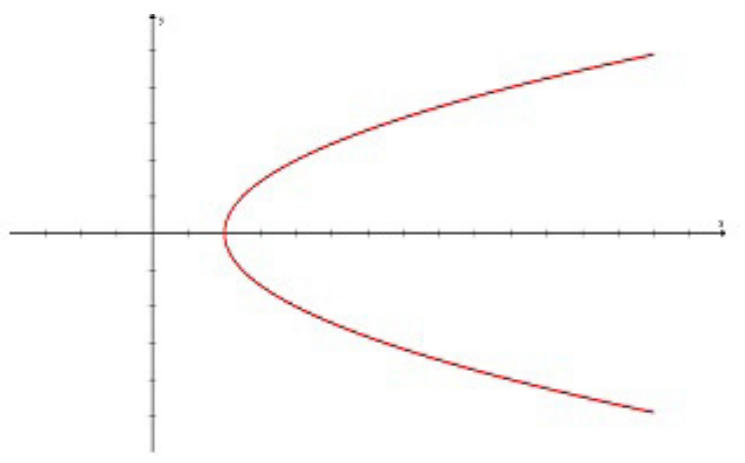

Las gráficas propuestas atienden a diversas situaciones que cotidianamente son generadoras de errores en la determinación de funciones (según resultados de investigaciones ya citadas), entre las que se encuentran la aplicación del criterio de la recta vertical, la generalización de que toda parábola es una función y la asociación de gráfica continua para ser función. De acuerdo con esta creencia, una función debe estar representada por una gráfica regular y continua, con lo cual se excluyen de tajo muchas funciones definidas por intervalos.

El instrumento se aplicó durante la primera semana de clases del semestre y sirvió para diagnosticar el nivel de dominio adquirido por los estudiantes una vez finalizan su ciclo de formación de educación media. La aplicación de la prueba comprendió 120 minutos. Los datos recolectados fueron digitalizados y exportados a los softwares Statgraphics Centurion XVII y Atlas/ti 7.0. A través del primero se efectuó un análisis descriptivo de las características sociodemográficas de la muestra y con el segundo se realizó un análisis cualitativo de los argumentos suministrados por los estudiantes ante las gráficas suministradas.

\section{Recolección y análisis de datos}

Para analizar los datos suministrados por los estudiantes se consideró de forma individual cada respuesta argumentada como un dato, convirtiéndose

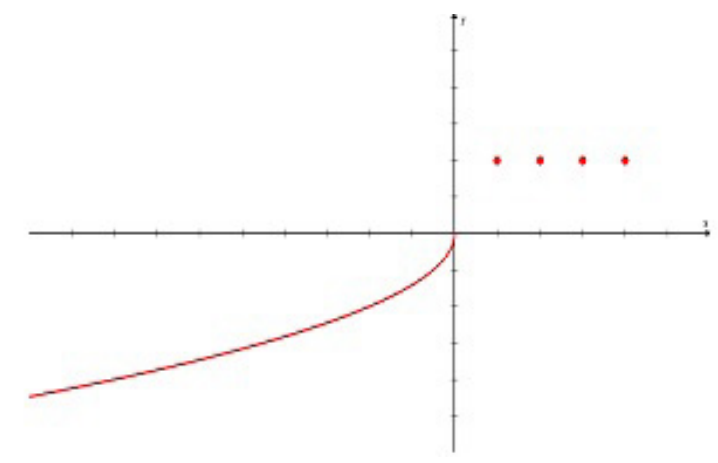

Figura 1. Ítem 1 del instrumento. Fuente: elaboración propia. 
cada argumento en una unidad de análisis. En consecuencia, el proceso indagatorio siguió la línea de la inducción analítica. Cada uno de los argumentos fue objeto de codificación (Strauss y Corbin, 2002), proceso mediante el cual se hace una interpretación teórica o conceptual sobre cada argumento y se relaciona con uno o varios códigos o etiquetas (Flick, 2004). Esta técnica se emplea con el fin de hacer emerger teoría y relaciones desde los datos. Una vez concluido el proceso se obtuvieron 280 unidades de análisis organizadas en las categorías conceptuales.

El proceso de codificación teórica abarca tres tipos de codificación: abierta, axial y selectiva. La codificación abierta permite identificar los fenómenos y se inicia con la conceptualización, que implica: a) reunir acontecimientos, sucesos u objetos similares bajo un encabezamiento que los clasifique basándose en una característica común; b) hacer una abstracción de los datos con el fin de descomponerlos en ideas, acontecimientos, incidentes y actos para luego darles una denominación que los represente o los substituya. La codificación axial tiene como propósito reagrupar los datos que conforman la categoría y relacionarlos con sus subcategorías con el fin de conseguir explicaciones más precisas y completas sobre los fenómenos. La codificación selectiva se refiere al proceso de integrar y refinar las categorías elegidas.

El proceso de codificación se llevó a cabo a través del software Atlas.ti, en el cual cada una de las 280 respuestas dadas por los estudiantes fue sometida al proceso de codificación abierta y axial. En este proceso cada segmento representa un argumento de interés para la investigación. El resultado del proceso de codificación teórica es el sistema de categorías emergentes, que representa un listado de códigos con la categoría respectiva a la que pertenece. Este sistema constituye la base del análisis.

Para efectos del análisis, a través del programa Atlas.ti, un par de números anteceden a las citas o fragmentos, el primero hace referencia al gráfico (1 o 2) que fue sometido a la interpretación por parte del estudiante, el segundo corresponde al número de la interpretación dentro del conjunto, así por ejemplo el número 2:37 para una cita significa la interpretación número 37 realizada por un estudiante al ejercicio número 2. Para el caso de los códigos, los números que están a la derecha tienen el siguiente significado: el primero el número de citas asociadas y el segundo el número de códigos relacionados.

\section{Resultados}

La tabla 2 muestra la distribución de las categorías y subcategorías conceptuales que emergen del análisis cualitativo a partir de la codificación abierta y axial. De manera global se observa el predominio de ciertas variaciones conceptuales y el empleo de los registros semióticos al identificar el concepto de función. Prevalece el concepto de par ordenado, con aproximadamente el 27,0 \% de los casos, y la articulación del registro gráfico al escrito al evaluar el concepto por parte del estudiante. El concepto de continuidad, aparece en segundo lugar, con una representación del 20,7 $\%$ de los casos. Dentro de los registros semióticos el pensamiento variaciones es percibido por 9 estudiantes.

En cuanto al manejo de conceptos, se puede observar que no se establecen con absoluta claridad. Existen varias vías para la comprensión del concepto, y las aproximaciones conceptuales se observaron en un número importante de estudiantes, aproximadamente el 16,0 \% de los casos. El apoyo en el concepto de relación es fundamental en esta categoría y se observó en aproximadamente el $10,0 \%$ de las unidades analizadas. Las deficiencias conceptuales no alcanzaron un nivel significativo, pues tan solo aparecieron en menos del $4,0 \%$ de los casos y están relacionadas con confusiones entre tipos de funciones y la confusión de rectas horizontales con las rectas verticales en el plano cartesiano.

A continuación, se presenta el análisis cualitativo de cada una de las subcategorías y categorías siguiendo el orden inductivo mostrado en la tabla 2. 
Tabla 2. Sistema de categorías emergentes a partir del proceso de codificación abierta.

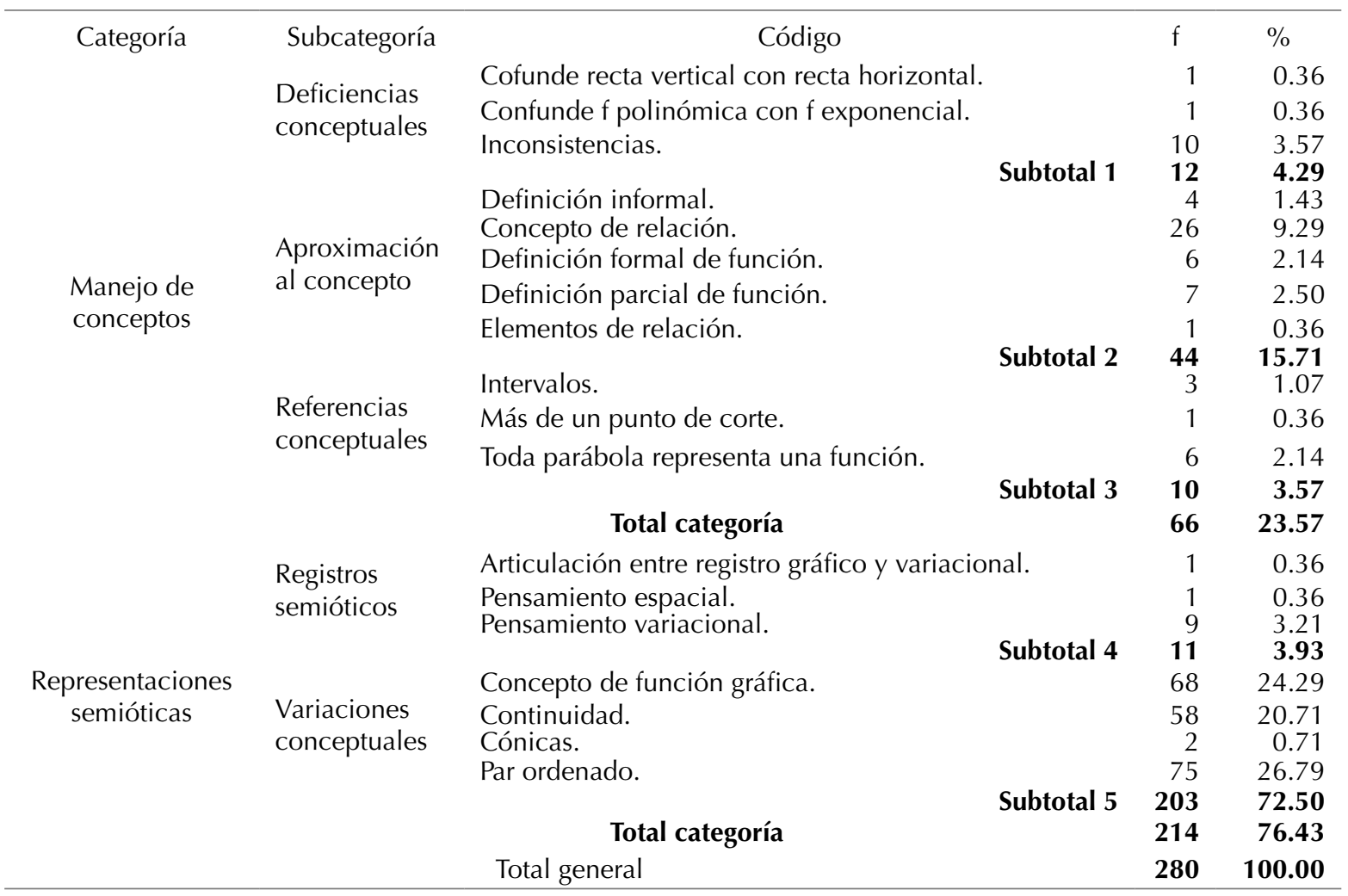

Fuente: elaboración propia.

En primer lugar, resalta un conjunto de deficiencias conceptuales (figura 2) representado por todos aquellos argumentos que carecen de dominio adecuado del concepto mediante la aplicación del pensamiento métrico y geométrico. Se hace alusión a conceptos equívocos que evidencian la falta de conocimiento con respecto a la noción del término función. Entre los argumentos para sustentar la presencia de una función en las gráficas presentadas se pueden señalar los siguientes:

Posee unas medidas o un rango en un plano cartesiano... [1:13].

Es una función exponencial [1:9].

Si se traza una línea horizontal y esta solo toca un punto, es una función [1:23].

En los ejemplos se advierte que el estudiante se apropia de expresiones inconsistentes en relación con el término. Tal es el caso de la expresión el uso de la recta horizontal, que evidencia deficiencias conceptuales. El estudiante recuerda un método gráfico para determinar funciones, que consiste en el trazo de una línea que debe tocar la gráfica en un único punto. El recuerdo de este método, sumado a la generalización de que toda parábola es función, Ileva a relacionar equívocamente la orientación de la línea recta de prueba como una recta horizontal, puesto que de esa forma satisfacen las dos gráficas y sus concepciones. Asimismo, se observan deficiencias en cuanto a los pensamientos aritmético y variacional, puesto que para algunos estudiantes cualquier expresión algebraica que tenga exponentes es una función exponencial.

Sin embargo, no todos los argumentos evidencian carencia absoluta de la noción del término. Por otra parte, en efecto, resaltan los argumentos que muestran una aproximación conceptual, si bien parcial, al 


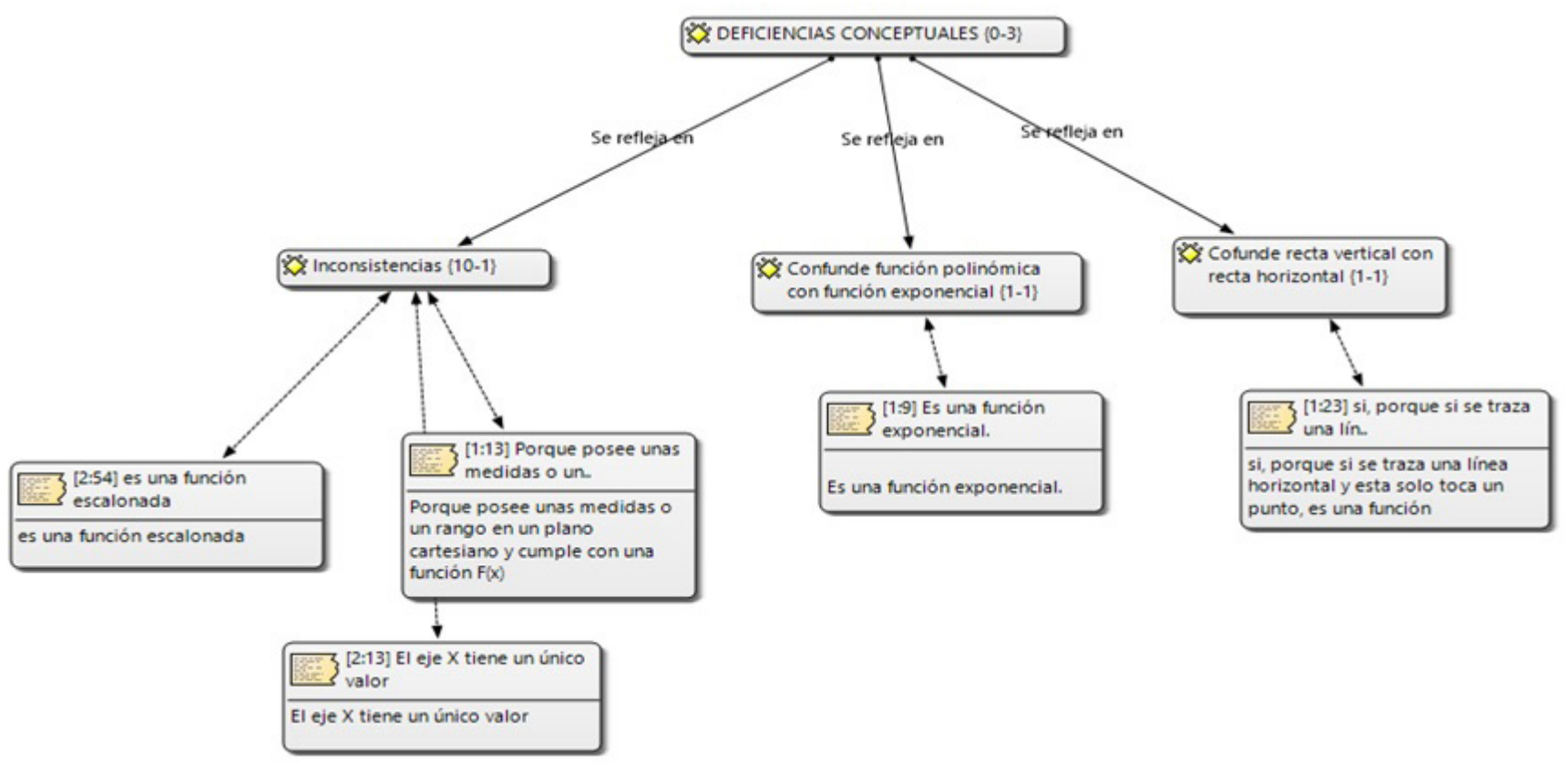

Figura 2. Deficiencias conceptuales.

Fuente: elaboración propia.

término función. La figura 3 muestra las tendencias encontradas entre los estudiantes que vislumbran una aproximación conceptual de función. En algunos casos esta aproximación está asociada al uso de formas coloquiales del lenguaje, mientras que en otros se utilizan algunos conceptos comúnmente considerados en una definición formal.

También se cuentan casos de estudiantes que utilizan el concepto de relación para evaluar si la gráfica representa o no función:

Al utilizar la prueba de la recta vertical se puede apreciar que corta en dos puntos. Por lo tanto es una relación y no una función [1:37].

Para cada una de las abscisas hay al menos más de una ordenada [1:5].

Entre estos estudiantes prevalece la noción de asociación entre los elementos de dos conjuntos como argumento en la demostración de su comprensión del término, con apoyo bien sea en una inspección visual de los dos conjuntos o en el uso de otras técnicas gráficas, como la de la recta vertical.
También se encuentran argumentos que ofrecen una aproximación informal al concepto sobre la base del número de imágenes asociadas con los elementos del dominio. Veamos un ejemplo:

Algunos puntos del eje tienen una y solo una imagen en el eje $Y, y$ ninguno de estos tiene 2 o más... [2:14].

Esta noción se ancla a la condición de imagen única en la correspondencia, lo que evidencia una mayor aproximación al concepto.

Aparecen también otros argumentos que intentan sustentarse a partir del uso de elementos constitutivos de su definición, como los que siguen:

En este caso el dominio tiene solo un elemento del codominio [2:17].

Para cada uno de los valores de $\mathrm{X}$ le corresponde un único valor en $\mathrm{Y}$ [2:37].

En ellos se aplican elementos distintivos en la definición, como los términos domino y codominio, 


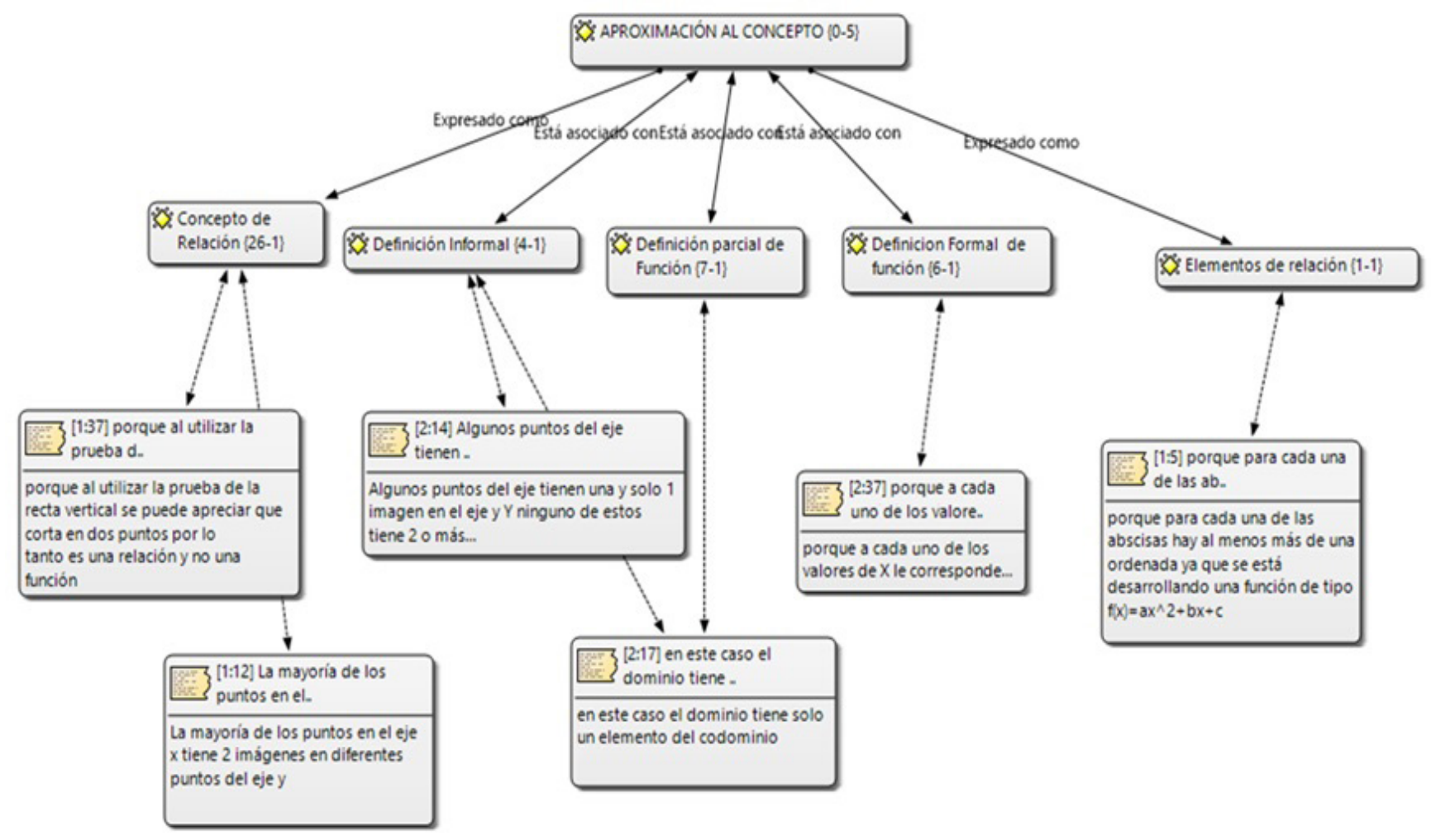

Figura 3. Aproximación al concepto.

Fuente: elaboración propia.

y la referencia algebraica a los conjuntos de partida y de llegada como $X$ y $Y$.

En este rango de interpretaciones, se evidencia el uso constante de referencias conceptuales asociadas al término función. Bajo esta categoría, que refiere la figura 4, se agrupan aquellos argumentos que los estudiantes utilizan como referentes teóricos para identificar una función, es decir, razones diversas que han ido acumulando a través de su proceso formativo, algunos como fruto de la búsqueda abreviada de procesos que desembocan en la generación de concepciones. Argumentos de este tipo son los siguientes:

Una función tiende a tener una parábola en el eje, y acá se forma una semiparábola [2:57].

Es una función, ya que en la representación del plano cartesiano se ve notoriamente la parábola [1:14].
Como se observa, en las explicaciones de estos estudiantes aparece la generalización de que toda parábola es una función, independientemente de cuál sea su eje principal. Asimismo, se destacan opiniones basadas el criterio de la recta vertical y en la asociación del número de puntos de corte de dicha recta sobre la trayectoria de la gráfica:

La recta vertical corta en dos puntos, por eso no es una función [1:4].

Entre las respuestas de los estudiantes se cuenta la adopción de representaciones semióticas para abordar este concepto. Bajo este esquema, que se demuestra en la figura 5, se agrupan aquellos argumentos en que los estudiantes recurren al uso independiente del pensamiento variacional: 


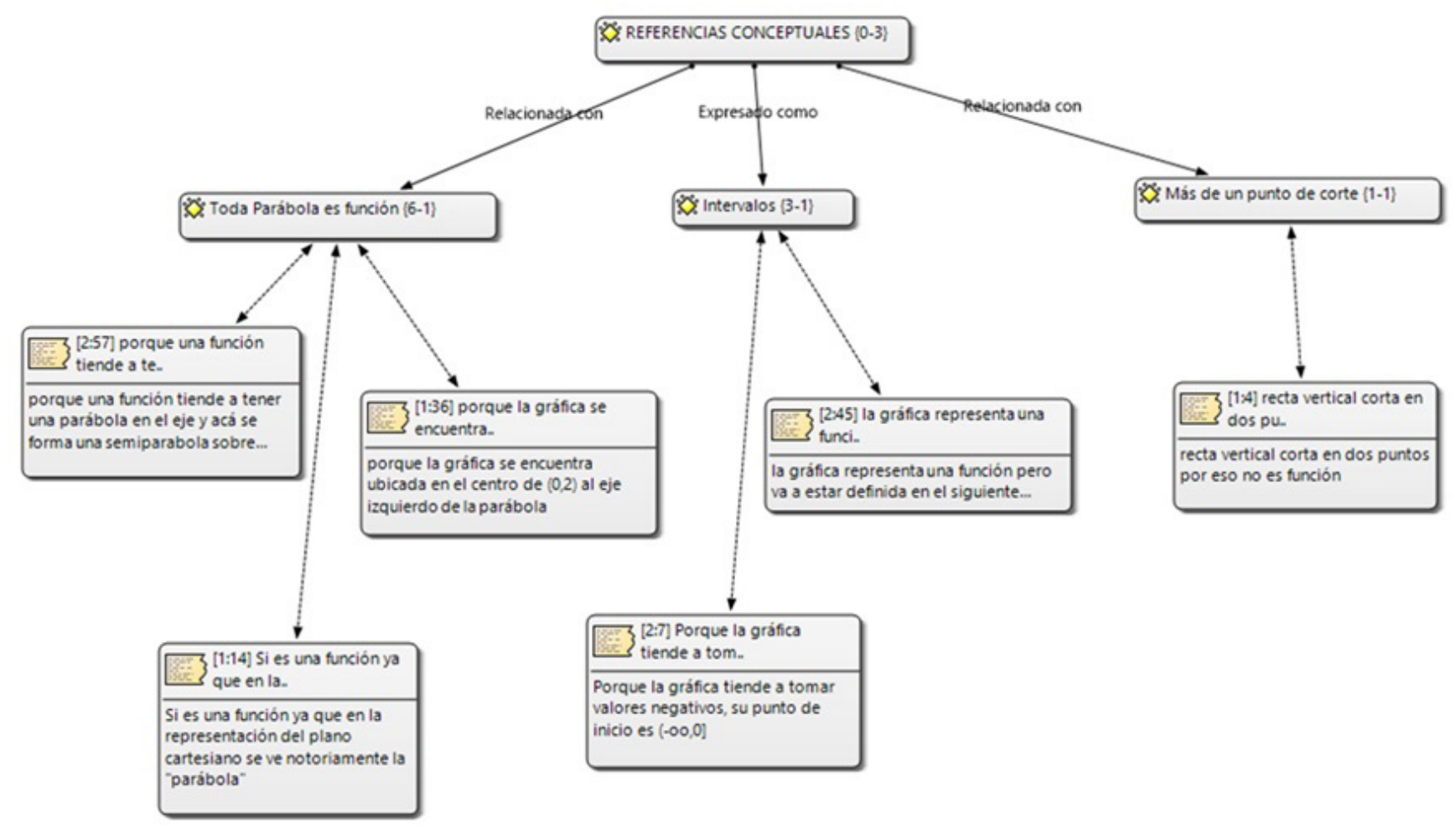

Figura 4. Referencias conceptuales.

Fuente: elaboración propia.

No es función porque al aplicar la prueba de la recta vertical $\mathrm{x}=5$ tiene dos imágenes en $\mathrm{Y}$ [1:32].

No es función porque el dominio se relaciona dos veces con el rango [1:10].

Lo mismo ocurre con el pensamiento espacial, que se intenta articular a partir de la gráfica realizando el cambio de registro para proponer expresiones algebraicas que la representen mejor:

Es una función porque para cada una de las abscisas hay al menos más de una ordenada, ya que se está desarrollando una función de tipo $f_{(x)}=a x^{2}+b x+c$, que al graficarla es una parábola [1:5].

Como se evidencia, los estudiantes ofrecen argumentos mediamente ciertos o errados, pero con ellos pretenden dar respuesta a la propuesta del instrumento.
Por otra parte, se evidencia en otros estudiantes la articulación de registros de representación semióticas, yendo del registro gráfico al registro variacional. Un ejemplo se encuentra en esta respuesta:

Es función porque está en función de $x=2$ cuando $y \rightarrow \alpha[1: 6]$.

En orden creciente de abstracción nos encontramos con las variaciones conceptuales (figura 6), en las que se agrupan todas las creencias o concepciones que poseen los estudiantes en torno al tema de funciones. En este conjunto de variaciones resaltan las opiniones de los estudiantes mediante las que expresan la necesidad de apoyarse en el concepto de par ordenado y lo visualizan como la relación entre los elementos de dos conjuntos llamados dominio y codominio (v.g.: Para cada dominio hay un codominio único [1:18]). 


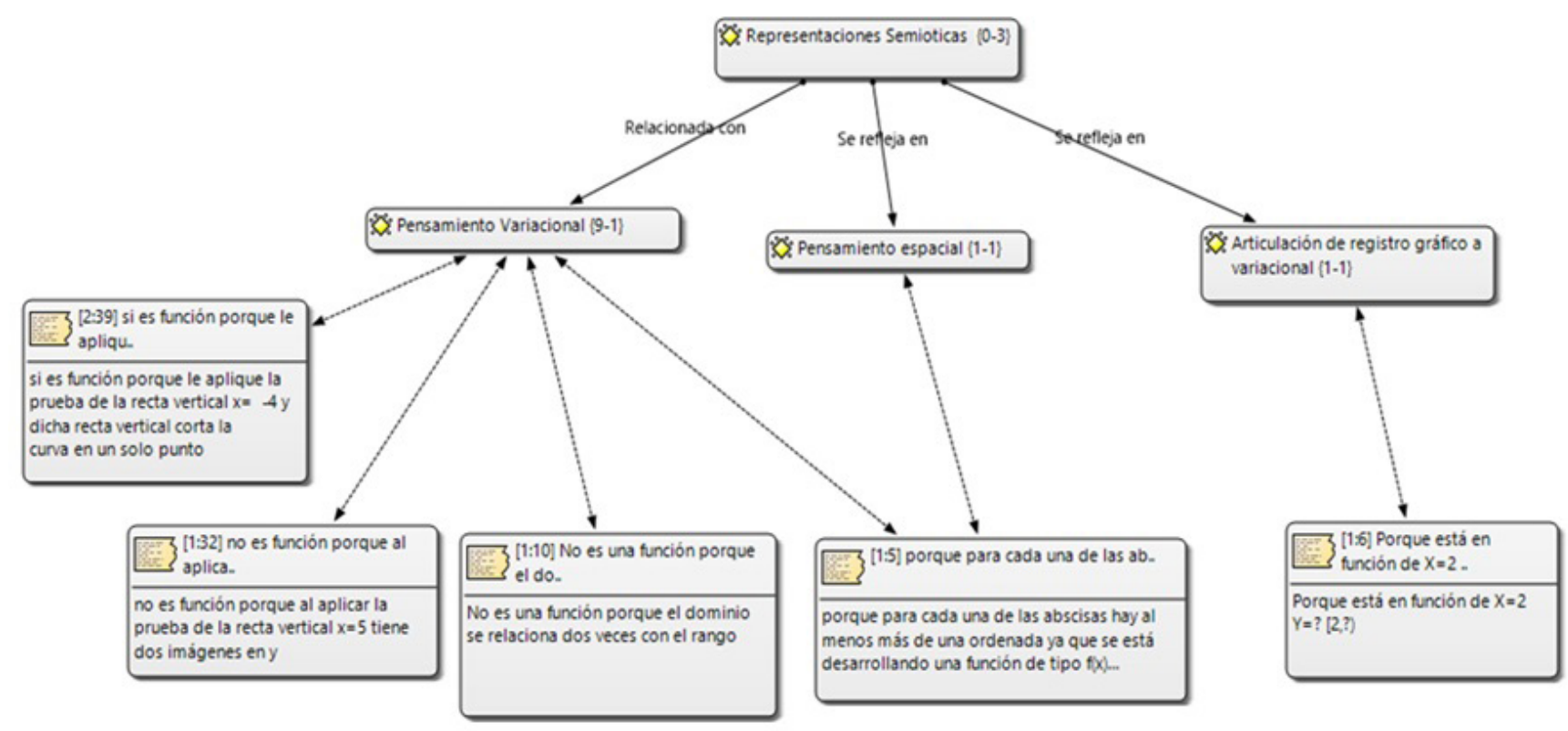

Figura 5. Representaciones semióticas.

Fuente: elaboración propia.

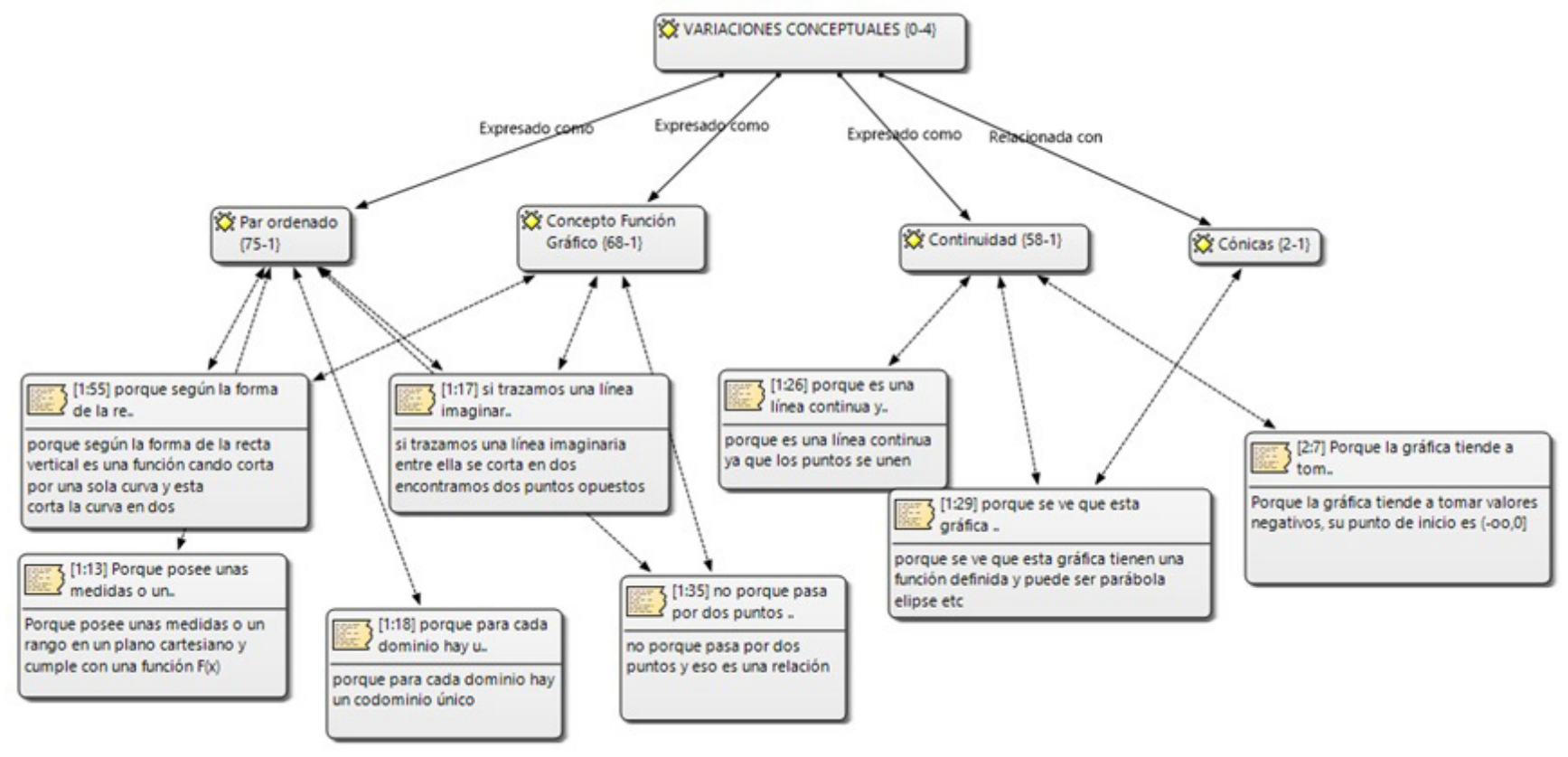

Figura 6. Variaciones conceptuales.

Fuente: elaboración propia. 
Por otra parte, se encuentran los estudiantes que apoyan su argumentación en el uso del registro gráfico a través de la prueba de la recta vertical, cuya aceptación o rechazo depende del número de puntos de corte de dicha recta con la trayectoria de la gráfica. Se evidencia en esta concepción la articulación con el registro gráfico pero con el uso del diagrama sagital, donde los puntos de corte representan el número de correspondencias que tiene el elemento del dominio. Otro criterio al que recurren los estudiantes es decidir a partir del registro gráfico y apoyados en la concepción de continuidad (Es una línea continua ya que los puntos se unen [1:26]). Según estas opiniones, una gráfica de función debe estar representada en el plano cartesiano por una línea (recta o curva) ininterrumpida y muy seguramente tomar forma de elipse o parábola (Se ve que esta gráfica tienen una función definida y puede ser parábola, elipse, etc. [1:29]). Bajo esta concepción de función se excluyen las funciones definidas a tramos o las funciones cuyo dominio sean los números naturales o los números enteros.

Como se puede observar, este nivel de clasificación pone de manifiesto muchas de las dificultades conceptuales que han ido surgiendo a medida que se ha afinado el concepto de función con el paso del tiempo. Muchas de las dificultades entendidas como concepciones (conocimiento local) se evidencian en este subnivel y en algunos casos son propiciadas por el docente, que aborda los detalles del concepto de una manera excesivamente informal y con cierta simplicidad del criterio, dejando por el camino muchos detalles indispensables para afinar el concepto.

\section{Discusión y Conclusiones}

De acuerdo con Vergnaud (1990), la operacionalidad de un concepto debe ser experimentada por medio de situaciones variadas. El profesor, por tanto, debe proponer una gran variedad de conductas y de esquemas para hacer comprender al estudiante en qué consisten conceptos complejos como los de razón, número o función.
De acuerdo con los resultados obtenidos, los estudiantes han sido expuestos a lo largo de su formación preuniversitaria al concepto de función. También se evidencia a través de sus conductas y argumentos que logran aproximarse en parte al término. Sin embargo, no puede afirmarse que en general manejen argumentos claros y contundentes, sino que echan mano de un conjunto de elementos que actúan como referentes conceptuales para la comprensión del término y su posterior manejo en las aplicaciones a otros contextos.

El estudio de las distintas aproximaciones al término función ha dejado claro que se trata de un elemento básico en el estudio del cálculo, quien posee la complejidad de otros términos matemáticos y que solo pueden ser comprendido a partir del manejo de campos conceptuales que proporcionen estructuras cognitivas y situaciones que contribuyan a su entendimiento. Los conceptos matemáticos, en efecto, se aprehenden mejor en consonancia con la teoría de los campos conceptuales, entendidos como un conjunto de situaciones cuyo tratamiento implica el manejo de nociones y teoremas que permiten analizar situaciones de la vida real como tareas matemáticas, lo cual se asocia con el aprendizaje significativo (Vergnaud, 1990, 2007).

Las dificultades observadas en las respuestas proporcionadas por los estudiantes se pueden agrupar en dos grandes niveles, descritos así:

En primer lugar, quedan de manifiesto en el contexto analizado las deficiencias en el manejo del concepto de función que los estudiantes recién graduados del sistema de educación media presentan al ingresar a la universidad. No existe, en efecto, un manejo conceptual claro en torno al término función. Se evidencia que existe un conocimiento relacional, pero no exacto acerca del concepto. Los estudiantes toman algunos elementos como referentes y se apoyan en otros conceptos algebraicos y geométricos para lograr una aproximación al concepto

En segundo término, las dificultades encontradas en las distintas representaciones semióticas 
siguen siendo un asunto que debe ser objeto de reflexión en la enseñanza de la matemática, especialmente en lo que concierne a la lectura e interpretación de gráficos. La investigación ha demostrado que las lecturas que los estudiantes dan en torno a la presentación de casos resultan en gran parte erradas. Por ello, tal y como señala Hitt (2003a) en las aulas de clase es preciso promover la articulación entre representaciones en las que se pongan en práctica la traslación entre diversos registros de representación semiótica.

Los resultados obtenidos concuerdan con la teoría de Duval (1999), que considera que el cambio de registros semióticos en el aprendizaje de conceptos matemáticos es una tarea progresiva que requiere de reforzamiento y contraste específico. Cuando se trata de nociones complejas, es preciso que los conceptos matemáticos se consoliden a lo largo del tiempo en las asignaturas afines dentro de un determinado currículo. Esto permitirá la construcción cada vez más acabada de los conceptos matemáticos y su aplicación a la hora de necesitar discriminar situaciones en los que se aplicarían (o no) y su adecuada interpretación en distintos contextos.

Por último, cabe destacar que existe un factor intangible, pero con efecto sobre los resultados y es la motivación e interés que tenga el estudiante por el tema en estudio, ya que, de no existir dicho interés, los resultados nunca serán favorables (situación observada en un pequeño grupo de estudiantes que se mantuvieron apáticos a la actividad).

\section{Agradecimientos}

El presente artículo de investigación forma parte de un estudio titulado "Efecto de la implementación de las representaciones semióticas alrededor del concepto de función en estudiantes de primer semestre de la Facultad de la Ingeniería", financiado por el Fondo de Investigación de la Universidad Francisco de Paula Santander (FINU), según contrato 021-2015.

\section{Referencias Bibliográficas}

Artigue, M. (1995). La enseñanza de los principios del cálculo: problemas epistemológicos, cognitivos y didácticos. En: M. Artigue, R. Douady, L. Moreno y P. Gómez (eds.) Ingeniería didáctica en educación matemática. Un esquema para la investigación y la innovación en la enseñanza y el aprendizaje de las matemáticas (pp. 97-140). Bogotá: Grupo Editorial Iberoamérica.

Blumer, H. (1992). Symbolic Interactionism: Perspective and Method. Oakland: University of California Press.

Cantoral, R. y Farfán, R. (1998). Pensamiento y lenguaje variacional en la introducción al análisis. Epsilon, 42(14), 353-369.

Carlile, P. y Christensen, C. (2011). Ciclos de la construcción de teorías en la investigación sobre gerencia. Trad. de J. Malave. Recuperado de: http://www.iesa.edu.ve/FILES MediaBroker/Public/ PDF/1048.pdf

Douady, R. (1986). Jeux de cadres et dialectique outil-objet. Recherches en didactique des mathématiques, 7(2), 5-31

Douady, R. (1995). La ingeniería didáctica y la evolución de su relación con el conocimiento. En: M. Artigue, R. Douady, L. Moreno y P. Gómez (eds.). Ingeniería didáctica en educación matemática. Un esquema para la investigación y la innovación en la enseñanza y el aprendizaje de las matemáticas (pp. 61-96). Bogotá: Grupo Editorial Iberoamérica.

Douady, R. (1996). Ingeniería didáctica y evolución de la relación con el saber en las matemáticas de collège-seconde. En: E. Barbin y R. Douady (eds.). Enseñanza de las matemáticas: Relación entre saberes, programas y prácticas. París: Topiques Éditions.

Dubinsky, E. (2002). Reflective abstraction in advanced mathematical thinking. En: D. Tall (ed.). Advanced Mathematical Thinking (pp. 95-126). Nueva York, Boston, Dordrecht, Londres, Moscú: Kluwer Academic Publisher. 
Duval, R. (1988). Graphiques et equations: L' Articulation de deux registres. Annales didactique et de sciencies cognitives, 1, 235-253.

Duval, R. (1993). Registres de représentation sémiotique et fonctionnement cognitif de la pensée. Annales de didactique et de sciences cognitives, 5, 37-65.

Duval, R. (2004). Semiosis y pensamiento humano. Registros semióticos y aprendizajes intelectuales. Trad. de M. Vega. 2a. ed. Cali: Universidad del Valle, Grupo de Educación Matemática.

Duval, R. (2006). Un tema crucial en la educación matemática: la habilidad para cambiar el registro de representación. La Gaceta de la Real Sociedad Matemática Española, 9(1), 143-168.

Echevarría, H. (2005). Los diseños de investigación y su implementación en educación. Rosario: Homo Sapiens Ediciones.

Eisenberg, T. (2002). Functions and Associated Learning Difficulties. En: D. Tall (ed.). Advanced Mathematical Thinking (pp. 95-126). Nueva York, Boston, Dordrecht, Londres, Moscú: Kluwer Academic Publisher.

Farfán, R. y García, M.A. (2005). El concepto de función: un breve recorrido epistemológico. En: J. Lezama, M. Sánchez y J.G. Molina (eds.). Acta latinoamericana de investigación en matemática educativa (RELIME), 18, 489-494.

Ferrari, M. (2001). Una visión socioepistemológica. Estudio de la función logaritmo. Tesis de maestría inédita. México: Cinvestav-IPN.

Flick, U. (2004). Introducción a la investigación cualitativa. 3a. ed. Madrid: Morata.

García, J.D. (2013). El concepto de función como una integración de los registros de representación. Tesis de maestría inédita. Medellín: Universidad Nacional de Colombia.

Garzón, D.A. (2015). Modelado de funciones desde el enfoque cognitivo de las representaciones semióticas. Tesis de maestría inédita. Medellín: Universidad de Antioquia.

Glasser, B.G., y Strauss, A.L. (1967). The Discovery of Grounded Theory: Strategies for Qualitative
Research. New Brunswick (EE. UU.): Aldine Transaction.

Hitt, F. (1994). Teachers' Difficulties with the Construction of Continuous and Discontinuous Functions. Focus on Lemming Problems in Mathematics, 16(4), 33-40.

Hitt, F. (1998). Difficulties in the Articulation of Different Representations Linked to the Concept of Function. Journal of Mathematical Behavior, 17(1), 123-134.

Hitt, F. (2000). Construcción de conceptos matemáticos y de estructuras cognitivas. En: Memorias de la XI Semana Regional de Investigación y Docencia en Matemáticas Universidad de Sonora. Sonora: Universidad de Sonora.

Hitt, F. (2003a). El concepto de infinito: obstáculo en el aprendizaje de límite y continuidad de funciones. En: E. Filloy, F. Hitt, C. Imaz, F. Rivera y S. Ursini (eds.). Matemática educativa: Aspectos de la investigación actual (pp. 91-111). México: Fondo de Cultura Económica.

Hitt,F.(2003b).Dificultadesenelaprendizajedelcálculo. Recuperadode:www.academia.edu/807014/ Dificultades en el aprendizaje del cálculo

Hitt, F. (2003c). El carácter funcional de las representaciones. Annales de didactique et de sciencies cognitives, 8, 255-271.

Hitt, F. y Torres, A. (1994). Visualizando las funciones con la PC. México: Grupo Editorial Iberoamérica.

Kaput, J.J. (1998). Representations, inscriptions, descriptions and learning: A kaleidoscope of windows. Journal of Mathematical Behavior, 17(2), 265-281.

Moreira, M.A. (2002). A teoria dos campos conceituais de Vergnaud, o ensino de ciências e a pesquisa nesta área. Investigações em Ensino de Ciências, 7(1), 7-29.

Ospina, D. (2012). Las representaciones semióticas en el aprendizaje del concepto función lineal. Tesis de maestría inédita. Manizales: Universidad Autónoma de Manizales.

Planchart, O. (2002). La visualización y la modelación en la adquisición del concepto de 
función. Tesis doctoral inédita. Cuernavaca: Universidad Autónoma del Estado de Morelos. Rojas, P.J. (2012). Articulación y cambios de sentido en situaciones de tratamiento de representaciones simbólicas de objetos matemáticos. Tesis doctoral inédita. Bogotá: Universidad Distrital Francisco José de Caldas.

Rojas, P.J. (2014). Articulación de saberes matemáticos: representaciones semióticas y sentidos. Bogotá: Comité Editorial Interinstitucional (CAIDE) - Universidad Distrital Francisco José de Caldas.

Ruthven, K. (1990). The influence of graphic calculator use on translation from graphic to symbolic forms. Educational Studies in Mathematics, 21(5), 431-450.

Sánchez, P.; Martínez, M.A. y Coronado, A. (2015). Una caracterización de la Competencia Matemática Representar: el caso de la función lineal. Amazonia Investiga, 4(7), 19-28.

Sierpinska, A. (1992). On understanding the notion of function. The concept of function: Aspects of epistemology and pedagogy, 25, 23-58.

Strauss, A. y Corbin, J. (2002). Bases de la Investigación cualitativa. Técnicas y procesamientos para desarrollar la teoría fundamentada. Medellín: Editorial Universidad de Antioquia.

Tall, D. (1992). The Transition to Advanced Mathematical Thinking: Functions, Limits, Infinity, and Proof. En: D.A. Grouws (ed.). Handbook of research on mathematics teaching and learning: A project of the National Council of Teachers of Mathematics (pp. 495-511). Nueva York: Macmillan.
Tall, D. (2002). The Psychology of Advanced Mathematical Thinking. En: D. Tall (ed.). $A d$ vanced Mathematical Thinking (pp. 3-21). Nueva York, Boston, Dordrecht, Londres, Moscú: Kluwer Academic Publisher.

Vergnaud, G. (1983). Multiplicative structures. En: R. Lesh y M. Landau (eds.). Acquisition of mathemtics concepts and processes (pp. 127174). Nueva York: Academic Press Inc.

Vergnaud, G. (1990). La théorie des champs conceptuels. Recherches en didactique des mathématiques, 10(2), 133-170.

Vergnaud, G. (2007). ¿En qué sentido la teoría de los campos conceptuales puede ayudarnos para facilitar aprendizaje significativo? Trad. C. de Caballera. Ponencia presentada en el $\checkmark$ Encuentro Internacional sobre Aprendizaje Significativo, celebrado en Madrid, 11-15 septiembre de 2006. Publicada en francés. Investigações em ensino de ciências, 12(2), 285-302.

Vinner, S. (1992). The function Concept as a Prototype for Problems in Mathematics Learning. En: E. Dubinsky y G. Harel (eds.). The Concept of Function: Aspects of Epistemology and Pedagogy (pp. 195-213). Washington, D.C.: Mathematical Association of America.

Vinner, S. (2002). The role of definitions in the teaching and learning of mathematics. En: D. Tall (ed.). Advanced Mathematical Thinking (pp. 65-81). Nueva York, Boston, Dordrecht, Londres, Moscú: Kluwer Academic Publisher. 